\title{
First-order electroweak phase transition powered by additional F-term loop effects in an extended supersymmetric Higgs sector
}

\author{
Shinya Kanemura ${ }^{1}$, Eibun Senaha ${ }^{2,3}$, Tetsuo Shindou $^{4}$ \\ ${ }^{1}$ Department of Physics, University of Toyama, 3190 Gofuku, Toyama 930-8555, Japan \\ 2 Korea Institute for Advanced Study, School of Physics, \\ 85 Hoegiro, Dongdaemun-gu, Seoul 130-722, Korea \\ 3 Physics Division, National Center for Theoretical Sciences, Hsinchu, Taiwan 300 \\ ${ }^{4}$ Division of Liberal Arts, Kogakuin University, 1-24-2 Shinjuku, Tokyo 163-8677, Japan
}

\begin{abstract}
We investigate the one-loop effect of new charged scalar bosons on the Higgs potential at finite temperatures in the supersymmetric standard model with four Higgs doublet chiral superfields as well as a pair of charged singlet chiral superfields. In this model, the mass of the lightest Higgs boson $h$ is determined only by the D-term in the Higgs potential at the tree-level, while the triple Higgs boson coupling for $h \mathrm{~h} h$ can receive a significant radiative correction due to nondecoupling oneloop contributions of the additional charged scalar bosons. We find that the same nondecoupling mechanism can also contribute to realize stronger first order electroweak phase transition than that in the minimal supersymmetric standard model, which is definitely required for a successful scenario of electroweak baryogenesis. Therefore, this model can be a new candidate for a model in which the baryon asymmetry of the Universe is explained at the electroweak scale.

PACS numbers: $\quad$ 14.80.Da, 12.60.Fr, 12.60.Jv
\end{abstract}

\section{INTRODUCTION}

It has been confirmed that the numbers of matter and anti-matter in our Universe are asymmetric. The recent observation suggests that the ratio for baryon-to-photon is given by $n_{b} / n_{\gamma} \simeq(5.1-6.5) \times 10^{-10}$ at the $95 \%$ CL [1], where $n_{b}$ is the difference in number density between baryons and anti-baryons and $n_{\gamma}$ is the number density of photons. Understanding the mechanism of generating the baryon asymmetry of the Universe has been one of the most important issues in particle physics and cosmology for a long time. In order to generate the baryon asymmetry from the baryon symmetric initial state, the following Sakharov's three conditions have to be satisfied [2]: 1) Baryon number nonconservation, 2) $\mathrm{C}$ and $\mathrm{CP}$ violation, 3) Departure from the thermal equilibrium.

The electroweak gauge theory can naturally satisfy the above three conditions. The baryon number nonconservation is realized by the sphaleron process at high temperature. The $\mathrm{C}$ violation can naturally occur in chiral gauge theories, and $\mathrm{CP}$ violating phases can remain in the matter sector after rephasing. Finally, the circumstance of thermal non-equilibrium can appear if the electroweak phase transition (EWPT) is of strongly first order. This scenario is often called the electroweak baryogenesis [3, 4]. The most attractive feature of this scenario would be its testability. The scenario is necessarily related to the dynamics of electroweak symmetry breaking, so that it can be directly tested by measuring the Higgs boson properties at collider experiments. Currently, Higgs boson searches are underway at the Tevatron and the LHC. We expect that the physics of electroweak symmetry breaking will be clarified in near future. Then the models of electroweak baryogenesis can be experimentally tested.
In the standard model (SM), it is found that the $\mathrm{CP}$ violation by the Cabibbo-Kobayashi-Maskawa matrix is quantitatively insufficient [5]. In addition, the requirement of sufficiently strong first order EWPT predicts the Higgs boson mass to be too small to satisfy the constraint from the LEP direct search results [6]. Therefore, in order to realize the baryon asymmetry of the Universe we need to consider an extension of the SM.

One of the viable models for successful electroweak baryogenesis would be the two Higgs doublet model (THDM) 7]. The extension of the Higgs sector can introduce additional $\mathrm{CP}$ violating phases, and the quantum effect of extra scalar bosons in the Higgs potential makes it possible to realize sufficiently strong first order EWPT without contradicting the LEP data for the mass of the Higgs boson. In Ref. 8], the connection between the first order EWPT and the triple coupling for the lightest SMlike Higgs boson $h$ (the $h h h$ coupling) has been clarified. In the model with sufficiently strong first order EWPT, the $h h h$ coupling constant significantly deviates from the SM prediction due to the same nondecoupling quantum effects of additional scalar bosons which make the first order EWPT strong. Such nondecoupling effects on the $h h h$ coupling constant have been studied in Ref. [9]. The scenario of electroweak baryogenesis by nondecoupling loop effects of extra bosons has also been applied in a $\mathrm{TeV}$ scale model where tiny neutrino masses, dark matter and the baryon asymmetry of the Universe may be simultaneously explained [10].

It would also be attractive to consider the scenario of electroweak baryogenesis in the model based on supersymmetry (SUSY). SUSY is a good candidate of new physics, which eliminates the quadratic divergence in the one-loop calculation of the Higgs boson mass. The lightest SUSY partner particle in SUSY models with the $\mathrm{R}$ 
parity can naturally be a candidate for the cold dark matter.

In the minimal supersymmetric SM (MSSM), there are many studies to realize the electroweak baryogenesis 11 13]. Currently, this scenario is highly constrained by the experimental data, especially the LEP Higgs mass bounds, leading to the tension between the lightest Higgs boson mass and the strength of the first order EWPT. Nevertheless, it is still viable for some specific mass spectrum. According to Ref. 12], the strong first order EWPT is possible if $m_{h} \lesssim 127 \mathrm{GeV}$ and $m_{\tilde{t}_{1}} \lesssim 120 \mathrm{GeV}$, where $h$ is the lightest Higgs boson and $\tilde{t}_{1}$ is the lightest stop. To satisfy the LEP bound on $m_{h}$, the soft SUSY breaking mass for the left-handed stop should be greater than $6.5 \mathrm{TeV}$. The most striking feature of this scenario is that the electroweak vacuum is metastable and the global minimum is a charge-color-breaking vacuum, where the lifetime of the electroweak vacuum is found to be longer than the age of the Universe.

The aforementioned tension in the MSSM baryogenesis can be relaxed by extending the Higgs sector. One of the possibilities is to add a gauge singlet field into the MSSM. So far, many studies on the electroweak baryogenesis have been done in such singlet-extended MSSMs; i.e., the Next-to-MSSM [14], the nearly MSSM or the minimal non-MSSM [15], the $U(1)^{\prime}$-extended MSSM [16], the secluded $U(1)^{\prime}$-extended MSSM [17, 18], and so on. In this class of the models, the strong first order EWPT can be induced by the trilinear mixing term of the doublet and the singlet fields appearing in the tree-level Higgs potential. If this is the case, the mass constraints on the lightest Higgs boson and the light stop would be alleviated significantly. In the singlet-extended MSSM, however, the vacuum structure is inevitably more complicated than the MSSM, giving rise to the unrealistic vacua in the large portion of the parameter space, especially electroweak baryogenesis-motivated scenario [14, 17].

In this Letter, we consider how the electroweak phase transition can be of sufficiently first order due to the nondecoupling effect of additional scalar bosons in another extended SUSY standard model, where a pair of extra doublet chiral superfields $H_{3}(Y=-1 / 2)$ and $H_{4}$ $(Y=+1 / 2)$ and a pair of charged singlet chiral superfields $\Omega_{1}(Y=+1)$ and $\Omega_{2}(Y=-1)$ are introduced in addition to the MSSM content [19]. Since the singlet fields are charged rather than neutral, they do not have the vacuum expectation values (VEVs) as long as the $U(1)_{\mathrm{EM}}$ is preserved. Therefore, at least at the tree level, there is no vacuum instability caused by the singlet field as opposed to the neutral singlet-extended MSSM as mentioned above. On top of this, this model may be motivated by SUSY extensions of the models with additional charged singlet fields in which neutrino masses are generated by radiative corrections [10, 20]. In the present model, apart from the model in which an additional neutral singlet chiral superfield is added to the MSSM, there is no tree-level F-term contribution to the mass of the lightest Higgs boson, but there can be large

\begin{tabular}{|c|c|c|c|c|c|c|}
\hline & Spin 0 & Spin 1/2 & \begin{tabular}{|l|l}
$\mathrm{SU}(3)$ \\
\end{tabular} & $\mathrm{SU}(2$ & $\mathrm{U}(1)_{Y}$ & $Z_{2}$ \\
\hline$Q_{i}$ & $\tilde{q}_{L}=\left(\begin{array}{l}\tilde{u}_{L} \\
\tilde{d}_{L}\end{array}\right.$ & $q_{L}=\left(\begin{array}{l}u_{L} \\
d_{L}\end{array}\right)$ & 3 & 2 & $+\frac{1}{6}$ & + \\
\hline$U_{i}^{c}$ & $\tilde{u}_{R}^{*}$ & $\bar{u}_{R}$ & $\overline{3}$ & 1 & $-\frac{2}{3}$ & + \\
\hline$D_{i}^{c}$ & $\tilde{d}_{R}^{*}$ & $\bar{d}_{R}$ & $\overline{3}$ & 1 & $+\frac{1}{3}$ & + \\
\hline$L_{i}$ & $\tilde{\ell}_{L}=\left(\begin{array}{l}\tilde{\nu}_{L} \\
\tilde{e}_{L}\end{array}\right.$ & $\ell_{L}=\left(\begin{array}{l}\nu_{L} \\
e_{L}\end{array}\right)$ & 1 & 2 & $-\frac{1}{2}$ & + \\
\hline$E_{i}^{c}$ & $\tilde{e}_{R}^{*}$ & $\bar{e}_{R}$ & 1 & 1 & +1 & $1+$ \\
\hline$H_{1}$ & $\Phi_{1}=$ & $\tilde{\Phi}_{1 L}=\left(\begin{array}{c}\tilde{\varphi}_{1 L}^{0} \\
\tilde{\varphi}_{1 L}^{-}\end{array}\right)$ & 1 & 2 & $-\frac{1}{2}$ & 1 \\
\hline $\mathrm{H}_{2}$ & $\Phi_{2}=\left(\begin{array}{l}\varphi_{2}^{+} \\
\varphi_{2}^{0}\end{array}\right.$ & $\tilde{\Phi}_{2 L}=\left(\begin{array}{c}\tilde{\varphi}_{2 L}^{+} \\
\tilde{\varphi}_{2 L}^{0}\end{array}\right)$ & 1 & 2 & $+\frac{1}{2}$ & $\uparrow$ \\
\hline$H_{3}$ & $\Phi_{3}=$ & $\tilde{\Phi}_{3 L}=\left(\begin{array}{l}\tilde{\varphi}_{3 L}^{0} \\
\tilde{\varphi}_{3 L}^{-}\end{array}\right)$ & 1 & 2 & $-\frac{1}{2}$ & \\
\hline$H_{4}$ & $\Phi_{4}=\left(\begin{array}{l}\varphi_{4}^{+} \\
\varphi_{4}^{0}\end{array}\right.$ & $\tilde{\Phi}_{4 L}=\left(\begin{array}{c}\tilde{\varphi}_{4 L}^{+} \\
\tilde{\varphi}_{4 L}^{0}\end{array}\right)$ & 1 & 2 & $+\frac{1}{2}$ & \\
\hline$\Omega_{1}$ & $\omega_{1}^{+}$ & $\overline{\tilde{\omega}}_{1}^{-}$ & 1 & 1 & +1 & \\
\hline$\Omega_{2}$ & $\omega_{2}^{-}$ & $\tilde{\omega}_{2}^{-}$ & 1 & 1 & -1 & \\
\hline
\end{tabular}

TABLE I: Property of chiral superfields (and their component fields) under the symmetries of the model.

one-loop corrections to the triple Higgs boson coupling due to the additional bosonic loop contribution [19]. We note that in the SUSY Higgs sector with four doublet chiral superfields [21], all the quartic interactions in the Higgs potential come from the D-term so that there are no large nondecoupling quantum effects on the $h h h$ coupling [19]. Similarly to the case of the non-SUSY THDM, these nondecoupling bosonic loop contributions can also make first order phase transition stronger. We here show that the EWPT can be of sufficiently strong first order in this model.

\section{MODEL}

We consider the model with the chiral superfields shown in Table 1 [19]. The symmetries of the model are standard gauge symmetries. In addition, we impose a discrete $Z_{2}$ symmetry for simplicity. Although the $Z_{2}$ symmetry is not essential for our discussion, the symmetry works for avoiding the flavor changing neutral current at the tree level 21 24]. Furthermore, we assume that there is the $\mathrm{R}$ parity in our model.

The superpotential is given by

$$
\begin{aligned}
W & =\left(y_{u}\right)^{i j} U_{i}^{c} H_{2} \cdot Q_{j}+\left(y_{d}\right)^{i j} D_{i}^{c} H_{1} \cdot Q_{j}+\left(y_{e}\right)^{i j} E_{i}^{c} H_{1} \cdot L_{j} \\
& +\lambda_{1} \Omega_{1} H_{1} \cdot H_{3}+\lambda_{2} \Omega_{2} H_{2} \cdot H_{4} \\
& -\mu H_{1} \cdot H_{2}-\mu^{\prime} H_{3} \cdot H_{4}-\mu_{\Omega} \Omega_{1} \Omega_{2} .
\end{aligned}
$$


The soft-SUSY-breaking terms are given by

$$
\begin{aligned}
\mathcal{L}_{\text {soft }} & =-\frac{1}{2}\left(M_{1} \tilde{B} \tilde{B}+M_{2} \tilde{W} \tilde{W}+M_{3} \tilde{G} \tilde{G}\right) \\
& -\left\{\left(\tilde{M}_{\tilde{q}}^{2}\right)_{i j} \tilde{q}_{L i}^{\dagger} \tilde{q}_{L j}+\left(\tilde{M}_{\tilde{u}}^{2}\right)_{i j} \tilde{u}_{R i}^{*} \tilde{u}_{R j}+\right. \\
& \left.\left(\tilde{M}_{\tilde{d}}^{2}\right)_{i j} \tilde{d}_{R i}^{*} \tilde{d}_{R j}+\left(\tilde{M}_{\tilde{\ell}}^{2}\right)_{i j} \tilde{\ell}_{L i}^{\dagger} \tilde{\ell}_{L j}+\left(\tilde{M}_{\tilde{e}}^{2}\right)_{i j} \tilde{e}_{R i}^{*} \tilde{e}_{R j}\right\} \\
& -\left\{\tilde{M}_{H_{1}}^{2} \Phi_{1}^{\dagger} \Phi_{1}+\tilde{M}_{H_{2}}^{2} \Phi_{2}^{\dagger} \Phi_{2}+\tilde{M}_{H_{3}}^{2} \Phi_{3}^{\dagger} \Phi_{3}+\right. \\
& \left.+\tilde{M}_{H_{4}}^{2} \Phi_{4}^{\dagger} \Phi_{4}+\tilde{M}_{+}^{2} \omega_{1}^{+} \omega_{1}^{-}+\tilde{M}_{-}^{2} \omega_{2}^{+} \omega_{2}^{-}\right\} \\
& -\left\{\left(A_{u}\right)^{i j} \tilde{u}_{R i}^{*} \Phi_{2} \cdot \tilde{q}_{L j}+\left(A_{d}\right)^{i j} \tilde{d}_{R i}^{*} \Phi_{1} \cdot \tilde{q}_{L j}+\right. \\
& +\left(A_{e}\right)^{i j} \tilde{e}_{R i}^{*} \Phi_{1} \cdot \tilde{\ell}_{L j} \\
& \left.+\left(A_{1}\right) \omega_{1}^{+} \Phi_{1} \cdot \Phi_{3}+\left(A_{2}\right) \omega_{2}^{-} \Phi_{2} \cdot \Phi_{4}+\text { h.c. }\right\} .
\end{aligned}
$$

From $W$ and $\mathcal{L}_{\text {soft }}$, the Lagrangian is constructed as

$$
\begin{aligned}
\mathcal{L}= & \mathcal{L}_{\text {kinetic }}+\mathcal{L}_{\text {gauge-matter }} \\
& -\left(\frac{1}{2} \frac{\partial^{2} W}{\partial \varphi_{i} \partial \varphi_{j}} \psi_{L i} \cdot \psi_{L j}+\text { h.c. }\right) \\
& -\left|\frac{\partial W}{\partial \varphi_{i}}\right|^{2}-\frac{1}{2}\left(g_{a}\right)^{2}\left(\varphi_{\alpha}^{*} T_{\alpha \beta}^{a} \varphi_{\beta}\right)^{2}+\mathcal{L}_{\text {soft }},
\end{aligned}
$$

where $\varphi_{i}$ and $\psi_{L i}$ are respectively scalar and fermion components of chiral superfields, and $T_{\alpha \beta}^{a}$ and $g_{a}$ represent generator matrices for the gauge symmetries and corresponding gauge coupling constants.

The scalar component doublet fields $\Phi_{i}$ are parameterized as

$$
\begin{aligned}
\Phi_{1,3} & =\left[\begin{array}{c}
\frac{1}{\sqrt{2}}\left(\varphi_{1,3}+h_{1,3}+i a_{1,3}\right) \\
\phi_{1,3}^{-}
\end{array}\right], \\
\Phi_{2,4} & =\left[\begin{array}{c}
\phi_{2,4}^{+} \\
\frac{1}{\sqrt{2}}\left(\varphi_{2,4}+h_{2,4}+i a_{2,4}\right)
\end{array}\right],
\end{aligned}
$$

where $\varphi_{i}$ are classical expectation values, $h_{i}$ are CP-even, $a_{i}$ are CP-odd and $\phi_{i}^{ \pm}$are charged scalar states. where $\varphi_{i}$ are classical expectation values, $h_{i}$ are CP-even, $a_{i}$ are $\mathrm{CP}$-odd and $\phi_{i}^{ \pm}$are charged scalar states. We use the effective potential method to explore the Higgs sector. At the tree level, the effective potential for the Higgs fields is given by

$$
\begin{aligned}
& V_{0}\left(\varphi_{1}, \varphi_{2}, \varphi_{3}, \varphi_{4}\right) \\
& =\sum_{a=1}^{4} \frac{1}{2} \bar{m}_{a}^{2} \varphi_{a}^{2}+\frac{1}{2}\left(B \mu \varphi_{1} \varphi_{2}+B^{\prime} \mu^{\prime} \varphi_{3} \varphi_{4}+\text { h.c. }\right) \\
& \quad+\frac{g^{2}+g^{\prime 2}}{32}\left(\varphi_{1}^{2}-\varphi_{2}^{2}+\varphi_{3}^{2}-\varphi_{4}^{2}\right)^{2} .
\end{aligned}
$$

Using the effective potential, the vacuum is determined by the stationary condition as

$$
\left.\frac{\partial V_{\mathrm{eff}}}{\partial \varphi_{i}}\right|_{\left\langle\varphi_{i}\right\rangle=v_{i}}=0
$$

We assume that the $Z_{2}$ odd Higgs bosons do not have the $\operatorname{VEVs}\left(v_{3}=v_{4}=0\right)$ for simplicity, and we set $\sqrt{v_{1}^{2}+v_{2}^{2}} \equiv v(\simeq 246 \mathrm{GeV})$ and introduce $\tan \beta=v_{2} / v_{1}$. At the tree level, $v_{3}=v_{4}=0$ is guaranteed by requiring the nonnegative eigenvalues of $\left(\partial^{2} V_{0} / \partial \varphi_{i} \partial \varphi_{j}\right)_{\varphi_{i, j}=0}(i, j=3,4)$, i.e.,

$$
\bar{m}_{3}^{2} \bar{m}_{4}^{2}-B^{\prime 2} \mu^{\prime 2} \geq 0, \quad \bar{m}_{3}^{2}+\bar{m}_{4}^{2} \geq 0 .
$$

In the following, we exclusively focus on the $\left(\varphi_{1}, \varphi_{2}\right)$ space.

For the $Z_{2}$ even scalar states, after the symmetry breaking we have five physical states as in the MSSM; i.e., two $\mathrm{CP}$-even $h$ and $H$, a CP-odd $A$ and a pair of charged $H^{ \pm}$scalar bosons. The tree level mass formulae for these scalar states coincide with those in the MSSM.

The mass eigenstates for the $Z_{2}$ odd charged scalar states $\phi_{1}^{\prime \pm}$ and $\phi_{2}^{\prime \pm}$ are obtained by diagonalizing the component fields of doublet scalar fields $\Phi_{3}$ and $\Phi_{4}$, and $\Omega_{1}^{ \pm}$ and $\Omega_{2}^{ \pm}$from the charged singlet scalar fields $\omega_{1}$ and $\omega_{2}$. Their field dependent masses are given by

$$
\begin{aligned}
& \bar{m}_{\phi_{1,2}^{\prime \pm}}^{2}=\frac{1}{2}\left[\bar{m}_{3}^{2}+\bar{m}_{4}^{2}+\frac{1}{2}\left(\left|\lambda_{1}\right|^{2} \varphi_{1}^{2}+\left|\lambda_{2}\right|^{2} \varphi_{2}^{2}\right) \mp \sqrt{D_{\phi^{\prime}}}\right], \\
& \bar{m}_{\Omega_{1,2}^{ \pm}}^{2}=\frac{1}{2}\left[\bar{m}_{+}^{2}+\bar{m}_{-}^{2}+\frac{1}{2}\left(\left|\lambda_{1}\right|^{2} \varphi_{1}^{2}+\left|\lambda_{2}\right|^{2} \varphi_{2}^{2}\right) \mp \sqrt{D_{\Omega^{ \pm}}}\right],
\end{aligned}
$$

where

$$
\begin{aligned}
\bar{D}_{\phi^{\prime \pm}}= & \left(\bar{m}_{3}^{2}-\bar{m}_{4}^{2}+\frac{1}{2}\left(\left|\lambda_{1}\right|^{2} \varphi_{1}^{2}-\left|\lambda_{2}\right|^{2} \varphi_{2}^{2}\right)\right. \\
& \left.-\frac{g^{2}-g^{\prime 2}}{4}\left(\varphi_{1}^{2}-\varphi_{2}^{2}\right)\right)^{2}+4\left|B^{\prime}\right|^{2}\left|\mu^{\prime}\right|^{2}, \\
\bar{D}_{\Omega^{ \pm}}= & \left(\bar{m}_{+}^{2}-\bar{m}_{-}^{2}+\frac{1}{2}\left(\left|\lambda_{1}\right|^{2} \varphi_{1}^{2}-\left|\lambda_{2}\right|^{2} \varphi_{2}^{2}\right)\right. \\
& \left.-\frac{g^{\prime 2}}{2}\left(\varphi_{1}^{2}-\varphi_{2}^{2}\right)\right)^{2}+4\left|B_{\Omega}\right|^{2}\left|\mu_{\Omega}\right|^{2} .
\end{aligned}
$$

Mass parameters in the above formulas are $\bar{m}_{3,4}^{2}=$ $\tilde{M}_{H_{3,4}}^{2}+\left|\mu^{\prime}\right|^{2}$ and $\bar{m}_{+,-}^{2}=\tilde{M}_{+,-}^{2}+\left|\mu_{\Omega}\right|^{2}$. The physical masses can be obtained by replacing $\varphi_{i}$ by $v_{i}$, the values at the vacuum. On the other hand, masses of the additional $Z_{2}$ odd neutral scalar bosons do not receive the contributions from the $\left|\lambda_{i}\right|^{2}$-terms, and only receive those from the D-term and the soft-SUSY-breaking terms. Their loop effect on the effective potential are small, so that we neglect them in our later calculations.

The field dependent masses of the $Z_{2}$ odd charginos are given by

$$
\begin{aligned}
\bar{m}_{\tilde{\chi}_{1,2}^{\prime \pm}}^{2}=\frac{1}{2}\left[\left|\mu^{\prime}\right|^{2}+\left|\mu_{\Omega}\right|^{2}\right. & \\
& \left.+\frac{1}{2}\left(\left|\lambda_{1}\right|^{2}\left|\varphi_{1}\right|^{2}+\left|\lambda_{2}\right|^{2}\left|\varphi_{2}\right|^{2}\right) \mp \sqrt{\bar{D}_{\tilde{\chi}}}\right],
\end{aligned}
$$


where

$$
\begin{aligned}
\bar{D}_{\tilde{\chi}}= & \left(\left|\mu^{\prime}\right|^{2}-\left|\mu_{\Omega}\right|^{2}+\frac{1}{2}\left(\left|\lambda_{1}\right|^{2}\left|\varphi_{1}\right|^{2}-\left|\lambda_{2}\right|^{2}\left|\varphi_{2}\right|^{2}\right)\right)^{2} \\
& +2\left|\lambda_{1}^{*} \mu_{\Omega} \varphi_{1}+\lambda_{2} \mu^{\prime *} \varphi_{2}\right|^{2} .
\end{aligned}
$$

The physical masses can be obtained by replacing $\varphi_{i}$ by $v_{i}$. Notice that the masses do not vanish even when the invariant mass parameters $\mu^{\prime}$ and $\mu_{\Omega}$ are taken to be zero.

Since it is known that radiative corrections on the Higgs sector are very important to study the EWPT, we here focus on the one-loop contribution. The vacuum at the one-loop level is also determined from Eq. (6) with the one-loop corrected effective potential. The one-loop correction to the effective potential at zero temperature is given by

$$
V_{1}\left(\varphi_{1}, \varphi_{2}\right)=\sum_{i} c_{i} \frac{\bar{m}_{i}^{2}}{64 \pi^{2}}\left(\ln \frac{\bar{m}_{i}^{2}}{M^{2}}-\frac{3}{2}\right)
$$

where $V_{1}$ is regularized in the $\overline{\mathrm{DR}}$-scheme, $c_{i}$ is the degrees of freedom of the species $i, M$ is a renormalization scale which will be set on $m_{t}^{\text {pole }}$.

For the zero temperature $T=0$, the one-loop corrected mass matrix for the $\mathrm{CP}$ even neutral bosons can be calculated from the effective potential. We here consider the simple case such that $B^{\prime}=B_{\Omega}=\mu^{\prime}=\mu_{\Omega}=0$ in order to switch off the mixing effects. By using the effective potential method, the MSSM-like CP even Higgs boson mass matrix $\left(M_{h}^{2}\right)_{i j}$ with the leading $\lambda_{1,2}^{4}$ contributions is given as

$$
\begin{aligned}
& \left(M_{h}^{2}\right)_{11}=m_{Z}^{2} c_{\beta}^{2}-B \mu \tan \beta+\frac{\lambda_{1}^{4} v^{2} c_{\beta}^{2}}{16 \pi^{2}} \ln \frac{m_{\Omega_{2}^{ \pm}}^{2} m_{\Phi_{2}^{\prime \pm}}^{2}}{m_{\tilde{\chi}_{1}^{\prime \pm}}^{4}}, \\
& \left(M_{h}^{2}\right)_{22}=m_{Z}^{2} s_{\beta}^{2}-B \mu \cot \beta+\frac{\lambda_{2}^{4} v^{2} 2_{\beta}^{2}}{16 \pi^{2}} \ln \frac{m_{\Omega_{1}^{ \pm}}^{2} m_{\Phi_{1}^{\prime \pm}}^{2}}{m_{\tilde{\chi}_{2}^{\prime \pm}}^{4}}, \\
& \left(M_{h}^{2}\right)_{12}=\left(M_{h}^{2}\right)_{21}=B \mu-m_{Z}^{2} c_{\beta} s_{\beta},
\end{aligned}
$$

where 1,2 are labeled as

$$
\begin{aligned}
& m_{\Phi_{1}^{\prime \pm}}^{2}=\bar{m}_{4}^{2}-\frac{m_{Z}^{2}-2 m_{W}^{2}}{2} c_{2 \beta}+\frac{\lambda_{2}^{2} s_{\beta}^{2}}{2} v^{2}, \\
& m_{\Phi_{2}^{\prime \pm}}^{2}=\bar{m}_{3}^{2}+\frac{m_{Z}^{2}-2 m_{W}^{2}}{2} c_{2 \beta}+\frac{\lambda_{1}^{2} c_{\beta}^{2}}{2} v^{2}, \\
& m_{\Omega_{1}^{ \pm}}^{2}=\bar{m}_{-}^{2}+\left(m_{Z}^{2}-m_{W}^{2}\right) c_{2 \beta}+\frac{\lambda_{2}^{2} s_{\beta}^{2}}{2} v^{2} \\
& m_{\Omega_{2}^{ \pm}}^{2}=\bar{m}_{+}^{2}-\left(m_{Z}^{2}-m_{W}^{2}\right) c_{2 \beta}+\frac{\lambda_{1}^{2} c_{\beta}^{2}}{2} v^{2}, \\
& m_{\tilde{\chi}_{1}^{\prime \pm}}^{2}=\frac{\lambda_{1}^{2} v^{2} c_{\beta}^{2}}{2} \\
& m_{\tilde{\chi}_{2}^{\prime \pm}}^{2}=\frac{\lambda_{2}^{2} v^{2} s_{\beta}^{2}}{2} .
\end{aligned}
$$

The renormalized mass of the lightest Higgs boson $h$ is

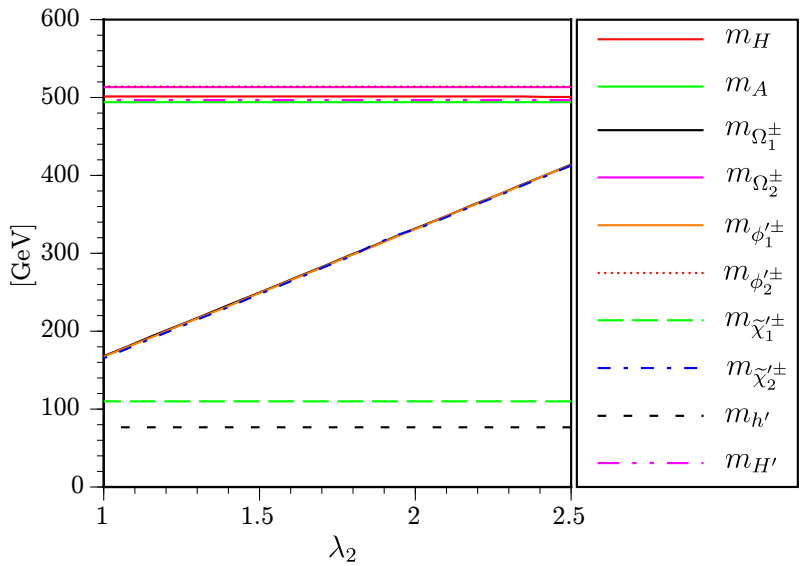

FIG. 1: The masses of the $Z_{2}$-odd scalars and $Z_{2}$-odd charginos as a function of $\lambda_{2}$. The masses of heavy $Z_{2}$-even neutral scalars $H$ and $A$ are also plotted. Model parameters are taken as them shown in Eq. (17). The $X_{t}$ dependence of these mass spectrum is negligible.

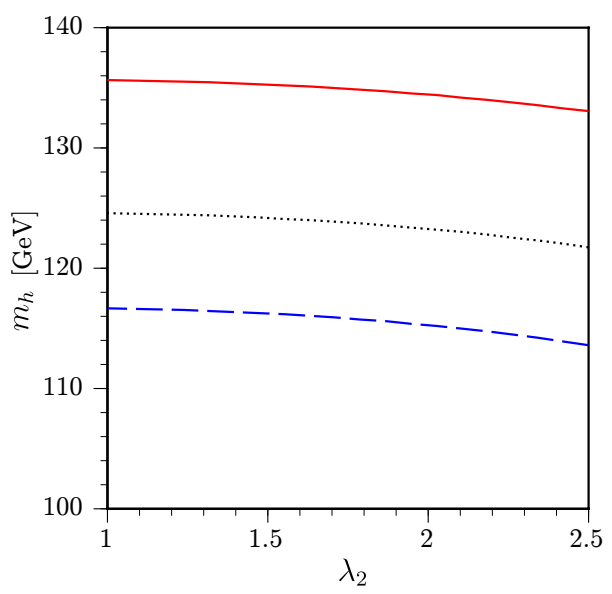

FIG. 2: The $Z_{2}$-even lightest Higgs boson mass as a function of $\lambda_{2}$. From the top to the bottom, $X_{t} / \tilde{M}_{\tilde{q}}=2.0,1.2$ and 0.6.

then calculated for $m_{A} \gg m_{Z}$ as

$$
\begin{aligned}
m_{h}^{2} & \simeq m_{Z}^{2} \cos ^{2} 2 \beta+(\text { MSSM-loop }) \\
& +\frac{\lambda_{1}^{4} v^{2} c_{\beta}^{4}}{16 \pi^{2}} \ln \frac{m_{\Omega_{2}^{ \pm}}^{2} m_{\Phi_{2}^{\prime \pm}}^{2}}{m_{\bar{\chi}_{1}^{\prime \pm}}^{4}}+\frac{\lambda_{2}^{4} v^{2} s_{\beta}^{4}}{16 \pi^{2}} \ln \frac{m_{\Omega_{1}^{ \pm}}^{2} m_{\Phi_{1}^{\prime \pm}}^{2}}{m_{\bar{\chi}_{2}^{\prime \pm}}^{4}}
\end{aligned}
$$

at the leading $\lambda_{1,2}^{4}$ contributions, where the one-loop contribution in the MSSM is mainly from the top and stop loop diagram 25].

Now we quantify the magnitude of the radiative corrections of the $Z_{2}$-odd particles on $m_{h}$. The input pa- 
rameters are fixed as follows.

$$
\begin{aligned}
\text { Tree }: & \tan \beta=3, m_{H^{ \pm}}=500 \mathrm{GeV} ; \\
\text { 1-loop }(\mathrm{MSSM}): & \tilde{M}_{\tilde{q}}=\tilde{M}_{\tilde{b}}=\tilde{M}_{\tilde{t}}=1000 \mathrm{GeV}, \\
& \mu=M_{2}=2 M_{1}=200 \mathrm{GeV}, \\
& A_{t}=A_{b}=X_{t}+\mu / \tan \beta ; \\
\text { 1-loop }\left(\Phi_{1,2}^{\prime \pm}, \Omega\right): & \lambda_{1}=2, \mu^{\prime}=\mu_{\Omega}=B_{\Omega}=B^{\prime}=0, \\
& \bar{m}_{+}^{2}=\bar{m}_{3}^{2}=(500 \mathrm{GeV})^{2}, \\
& \bar{m}_{-}^{2}=\bar{m}_{4}^{2}=(50 \mathrm{GeV})^{2} .
\end{aligned}
$$

We note that $m_{\Phi_{1}^{\prime \pm}}<m_{\Phi_{2}^{\prime \pm}}$ and $m_{\Omega_{1}^{ \pm}}<m_{\Omega_{2}^{ \pm}}$in this case. The mass spectrum of extra $Z_{2}$ odd charged scalars and charginos is displayed in Fig. 11. On this parameter set, $m_{\Phi_{1}^{\prime \pm}}^{2}, m_{\Omega_{1}^{ \pm}}^{2}$ and $m_{\tilde{\chi}_{2}^{\prime \pm}}^{2}$ get a significant contribution from $\lambda_{2}$. Then their masses become larger for the greater value of $\lambda_{2}$. Since the mass parameters $\bar{m}_{4}^{2}$ and $\bar{m}_{-}^{2}$ are taken to be small, large mass values of $m_{\Phi_{1}^{\prime \pm}}$ and $m_{\Omega_{1}^{ \pm}}$yield the large nondecoupling effects which can make the EWPT strongly first order.

Fig. 2 shows the predicted value of $m_{h}$ as a function of $\lambda_{2}$ varying $X_{t} / \tilde{M}_{\tilde{q}}=2.0,1.2$ and 0.6 from the top to the bottom. We can see that $m_{h}$ monotonically decreases as $\lambda_{2}$ increases, which is in contrast with the top/stop loop effects.

The coupling constants $\lambda_{1}$ and $\lambda_{2}$ are free parameters of the model. Its magnitude, however, is bounded from above by the condition that there is no Landau pole below the given cutoff scale $\Lambda$. As we are interested in the model where the first order EWPT is sufficiently strong, we allow rather larger values for these coupling constants, and do not require that the model holds until the grand unification scale. A simple renormalization group equation analysis tells us that for assuming $\Lambda=2 \mathrm{TeV}, 10 \mathrm{TeV}$ or $10^{2} \mathrm{TeV}$, the coupling constant can be taken to be at most $\lambda_{2} \sim 2.5,2.0$ or 1.5 , respectively. Above the cutoff scale $\Lambda$, the model may be replaced by a strongly coupled supersymmetric theory with UV completion as described by the scenario such as in the fat Higgs model [26].

\section{ELECTROWEAK PHASE TRANSITION}

The nonzero temperature effective potential is

$$
V_{1}\left(\varphi_{1}, \varphi_{2} ; T\right)=\sum_{i} c_{i} \frac{T^{4}}{2 \pi^{2}} I_{B, F}\left(\frac{\bar{m}_{i}^{2}}{T^{2}}\right),
$$

where $B(F)$ refer to boson (fermion) and $I_{B, F}$ take the form

$$
I_{B, F}\left(a^{2}\right)=\int_{0}^{\infty} d x x^{2} \ln \left(1 \mp e^{-\sqrt{x^{2}+a^{2}}}\right) .
$$

Since the minimum search using $I_{B, F}$ is rather timeconsuming, we will alternatively use the fitting functions of them that are employed in Ref. [13]. More explicitly,

$$
\tilde{I}_{B, F}\left(a^{2}\right)=e^{-a} \sum_{n=0}^{N} c_{n}^{b, f} a^{n}
$$

are used, where $c_{n}^{b, f}$ are determined by the least square method. For $N=40,\left|I_{B, F}\left(a^{2}\right)-\tilde{I}_{B, F}\left(a^{2}\right)\right|<10^{-6}$ for any $a$, which is sufficient in our investigation. Since the nonzero modes of the thermal effective potential give $T^{2}$ corrections to the 2-point self energy at high temperatures, we will resum them to make the perturbative analysis more reliable [27].

For an electroweak baryogenesis scenario to be successful, the sphaleron rate in the broken phase should be smaller than the Hubble constant. Conventionally, this condition is translated into

$$
\frac{v_{C}}{T_{C}}=\frac{\sqrt{v_{1}^{2}\left(T_{C}\right)+v_{2}^{2}\left(T_{C}\right)}}{T_{C}} \gtrsim \zeta,
$$

where $T_{C}$ is the critical temperature, $v_{C}$ is the Higgs $\mathrm{VEV}$ at $T_{C}$, and $\zeta$ is a $\mathcal{O}(1)$ parameter. To obtain $\zeta$ within a better accuracy, the sphaleron energy and zeromode factors of the fluctuations around the sphaleron must be evaluated. In the SM, the sphaleron energy is simply a function of the Higgs boson mass. As the Higgs boson becomes heavier, the sphaleron energy gets larger as well [28], leading to the smaller $\zeta$ 29]. In this model, on the other hand, $\zeta$ depends on more parameters. For simplicity, we here take $\zeta=1$, which is often adopted as a rough criterion in the literature.

In our analysis, $T_{C}$ is defined as the temperature at which the effective potential has the two degenerate minima. We search for $T_{C}$ by minimizing

$V_{\text {eff }}\left(\varphi_{1}, \varphi_{2} ; T\right)=V_{0}\left(\varphi_{1}, \varphi_{2}\right)+V_{1}\left(\varphi_{1}, \varphi_{2}\right)+V_{1}\left(\varphi_{1}, \varphi_{2} ; T\right)$,

where the field-dependent masses are modified by adding thermal corrections, and Eq. (20) is used in $V_{1}\left(\varphi_{1}, \varphi_{2} ; T\right)$.

In Fig. 3 $v_{C}$ and $T_{C}$ are plotted as a function of $\lambda_{2}$ in the light $h$ scenario $\left(X_{t} / \tilde{M}_{\tilde{q}}=0.6\right)$ : see Fig. 2. The sphaleron decoupling condition (21) can be fulfilled for $\lambda_{2} \gtrsim 1.6$ due to the nondecoupling effects coming from $\phi_{1}^{\prime \pm}$ and $\Omega_{1}^{ \pm}$.

We also evaluate $v_{C}$ and $T_{C}$ in the heavy $h$ scenario $\left(X_{t} / \tilde{M}_{\tilde{q}}=2.0\right)$ as shown in Fig. 4. The sphaleron decoupling condition can be satisfied for $\lambda_{2} \gtrsim 1.8$. Though the parameter region is a bit narrower than the light Higgs scenario, the lightest Higgs boson mass as large as $134 \mathrm{GeV}$ is still consistent with the decoupling condition.

\section{DISCUSSIONS}

The several comments on the current analysis are in order. 


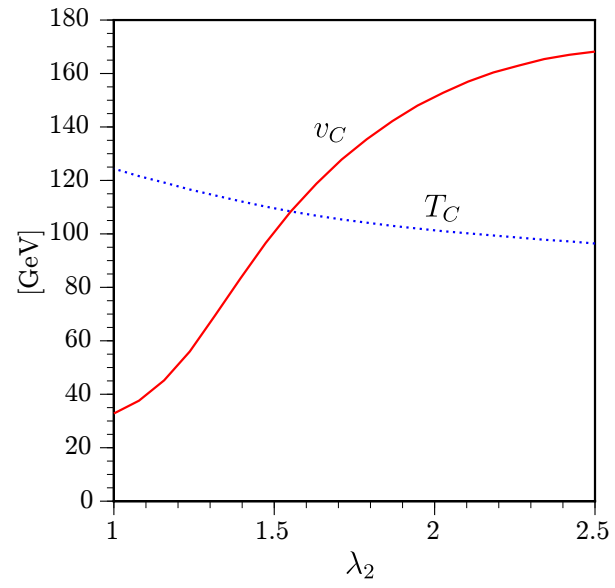

FIG. 3: $v_{C}$ and $T_{C}$ vs. $\lambda_{2}$ with $X_{t} / \tilde{M}_{\tilde{q}}=0.6$. The other input parameters are the same as in the Fig. 2. The sphaleron decoupling condition (21) can be satisfied for $\lambda_{2} \gtrsim 1.6$.

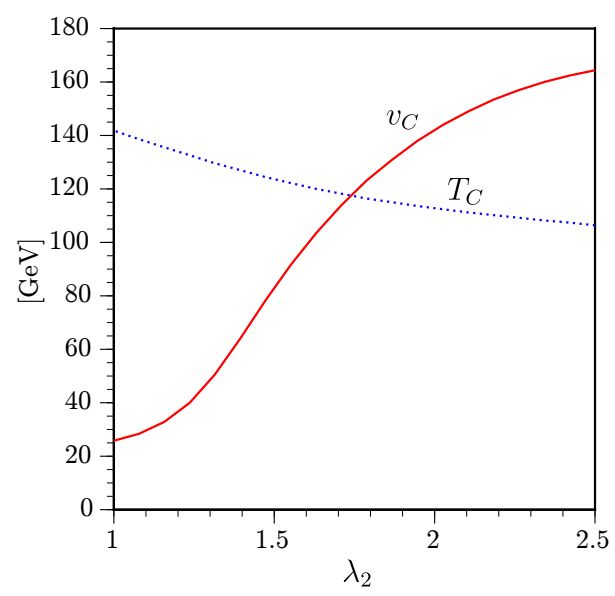

FIG. $4: v_{C}$ and $T_{C}$ vs. $\lambda_{2}$ with $X_{t} / \tilde{M}_{\tilde{q}}=2.0$. The other input parameters are the same as in the Fig. 2. The sphaleron decoupling condition (21) can be satisfied for $\lambda_{2} \gtrsim 1.8$.

- According to a study of the sphaleron decoupling condition in the MSSM, it is found that $\zeta \simeq 1.4$ [13] which is $40 \%$ stronger than one we impose in our analysis. The similar value may be obtained in this model as well. It should be emphasized, however, that even if we take $\zeta=1.4$ for the sphaleron decoupling condition, a feasible region exists for the relatively large $\lambda_{2}$, for example, $\lambda_{2} \gtrsim 2.2$ even in the heavy $h$ case. The cutoff scale $\Lambda$ is rather low in this case but still it is around the multi-TeV scale.

- In this model, the light stop scenario is one of the options for the successful electroweak baryogenesis. Same as the scenario in the MSSM, the strength of the first order EWPT can get enhanced if the (almost) right-handed stop is lighter than the top quark, enlarging the possible region.
- Similar to the usual MSSM baryogenesis scenario, the charginos and/or the neutralinos can play an essential role in generating the $\mathrm{CP}$ violating sources as needed for the bias of the chiral charge densities around the Higgs bubble walls. In addition to this, the $Z_{2}$ odd charginos $\widetilde{\chi}_{1,2}^{\prime \pm}$ may also do the job for the successful baryogenesis.

- Since $A_{1,2}$ and $\lambda_{1,2} \mu_{\Omega}$ are small in our parameter choices, the charge breaking does not occur at the tree level. In addition, the $Z_{2}$ symmetry is not broken spontaneously at the tree level, because $\bar{m}_{3}^{2}$, $\bar{m}_{4}^{2}$ and $B^{\prime} \mu^{\prime}$ we take here satisfy Eq. (77). The potential analysis beyond the tree level is out of scope in this Letter. It will be our future task.

- If the $Z_{2}$ symmetry is exact and unbroken after the electroweak symmetry breaking, the lightest $Z_{2}$ odd particle in our model can be a candidate of cold dark matter if it is electrically neutral, in addition to the lightest supersymmetric particle. If one of the extra neutral scalar bosons is the lightest $Z_{2}$ field, its phenomenological property and experimental constraints would be similar to those for the supersymmetric extension of the inert doublet model [30]. A neutralino from the extra doublets may also be a candidate for dark matter.

- Finally, we comment on the phenomenological predictions of this model. First of all, the nondecoupling effect of the extra $Z_{2}$ odd charged scalar bosons on the finite temperature effective potential is an essentially important feature of our scenario in order to realize strong first order phase transition. The same physics affects the triple Higgs boson coupling with a large deviation from the SM (MSSM) prediction as discussed in Ref. [8], Such deviation in the triple Higgs boson coupling can be $15-70 \%$ [9, 19], and we expect that they can be measured at the future linear collider such as the ILC or the CLIC. Second, in our model, in order to realize the nondecoupling effect large, the invariant parameters $\mu^{\prime}$ and $\mu_{\Omega}$ are taken to be small. Consequently, the masses of extra charginos are relatively as light as $100-300 \mathrm{GeV}$.

\section{CONCLUSIONS}

We have discussed the one-loop effect of new charged scalar bosons on the Higgs potential at finite temperatures in the supersymmetric standard model with four Higgs doublet chiral superfields as well as a pair of charged singlet chiral superfields. We have found that the nondecoupling loop effects of additional charged scalar bosons can make first order EWPT strong enough to realize successful electroweak baryogenesis. We, therefore, conclude that this model can be a new good candidate for a successful model where the baryon 
asymmetry of the Universe is explained at the electroweak scale. The detailed analysis for the collider phenomenology will be shown elsewhere.

\section{Acknowledgments}

The authors would like to thank Mayumi Aoki and
Kei Yagyu for useful discussions. This work was supported in part by Grant-in-Aid for Scientific Research (A) No. 22244031 [S.K.], Grant-in-Aid for Scientific Research in Priority Areas No. 22011007 [T.S.], and Grant-in-Aid for Scientific Research for Innovative Areas Nos. 23104006 [S.K.] and 23104011 [T.S.].
[1] K. Nakamura et al. [Particle Data Group], J. Phys. G 37 (2010) 075021.

[2] A. D. Sakharov, Pisma Zh. Eksp. Teor. Fiz. 5 (1967) 32.

[3] V. A. Kuzmin, V. A. Rubakov, M. E. Shaposhnikov, Phys. Lett. B155 (1985) 36; A. G. Cohen, D. B. Kaplan, A. E. Nelson, Nucl. Phys. B349 (1991) 727-742; Ann. Rev. Nucl. Part. Sci. 43 (1993) 27; M. Quiros, Helv. Phys. Acta 67 (1994) 451; V. A. Rubakov and M. E. Shaposhnikov, Usp. Fiz. Nauk 166 (1996) 493 [Phys. Usp. 39 (1996) 461].

[4] For reviews, see A. G. Cohen, D. B. Kaplan and A. E. Nelson, Ann. Rev. Nucl. Part. Sci. 43 (1993) 27; M. Quiros, Helv. Phys. Acta 67 (1994) 451; V. A. Rubakov and M. E. Shaposhnikov, Usp. Fiz. Nauk 166 (1996) 493 [Phys. Usp. 39 (1996) 461]; K. Funakubo, Prog. Theor. Phys. 96 (1996) 475; M. Trodden, Rev. Mod. Phys. 71 (1999) 1463; W. Bernreuther, Lect. Notes Phys. 591 (2002) 237.

[5] M. B. Gavela, P. Hernandez, J. Orloff and O. Pene, Mod. Phys. Lett. A 9 (1994) 795; M. B. Gavela, P. Hernandez, J. Orloff, O. Pene and C. Quimbay, Nucl. Phys. B 430 (1994) 382; P. Huet and E. Sather, Phys. Rev. D 51 (1995) 379; T. Konstandin, T. Prokopec and M. G. Schmidt, Nucl. Phys. B 679 (2004) 246.

[6] R. Barate et al. [LEP Working Group for Higgs boson searches and ALEPH and DELPHI and L3 and OPAL Collaborations], Phys. Lett. B565 (2003) 61-75.

[7] M. Joyce, T. Prokopec and N. Turok, Phys. Rev. D 53 (1996) 2930; Phys. Rev. D 53 (1996) 2958; J. M. Cline, K. Kainulainen and A. P. Vischer, Phys. Rev. D 54 (1996) 2451; J. M. Cline and P. A. Lemieux, Phys. Rev. D 55 (1997) 3873; L. Fromme, S. J. Huber and M. Seniuch, JHEP 0611 (2006) 038; A. Kozhushko, V. Skalozub, Ukr. J. Phys. 56 (2011) 431-442.

[8] S. Kanemura, Y. Okada and E. Senaha, Phys. Lett. B 606 (2005) 361.

[9] S. Kanemura, S. Kiyoura, Y. Okada, E. Senaha and C. P. Yuan, Phys. Lett. B 558 (2003) 157; S. Kanemura, Y. Okada, E. Senaha and C. P. Yuan, Phys. Rev. D 70 (2004) 115002.

[10] M. Aoki, S. Kanemura and O. Seto, Phys. Rev. Lett. 102 (2009) 051805; Phys. Rev. D80 (2009) 033007. M. Aoki, S. Kanemura, K. Yagyu, Phys. Rev. D83 (2011) 075016.

[11] M. S. Carena, M. Quiros and C. E. M. Wagner, Phys. Lett. B 380 (1996) 81; D. Delepine, J. M. Gerard, R. Gonzalez Felipe and J. Weyers, Phys. Lett. B 386 (1996) 183; P. Huet and A. E. Nelson, Phys. Rev. D 53 (1996) 4578; B. de Carlos and J. R. Espinosa, Nucl. Phys. B 503 (1997) 24; M. S. Carena, M. Quiros, A. Riotto, I. Vilja and C. E. M. Wagner, Nucl. Phys. B 503 (1997) 387; M. Aoki, A. Sugamoto and N. Oshimo, Prog. Theor. Phys. 98 (1997) 1325; M. Aoki, N. Oshimo and A. Sugamoto, Prog. Theor. Phys. 98
(1997) 1179; J. M. Cline, M. Joyce and K. Kainulainen, JHEP 0007 (2000) 018; M. S. Carena, J. M. Moreno, M. Quiros, M. Seco and C. E. M. Wagner, Nucl. Phys. B 599 (2001) 158; M. S. Carena, M. Quiros, M. Seco and C. E. M. Wagner, Nucl. Phys. B 650 (2003) 24; C. Lee, V. Cirigliano and M. J. Ramsey-Musolf, Phys. Rev. D 71 (2005) 075010; V. Cirigliano, M. J. RamseyMusolf, S. Tulin and C. Lee, Phys. Rev. D 73 (2006) 115009; T. Konstandin, T. Prokopec, M. G. Schmidt and M. Seco, Nucl. Phys. B 738 (2006) 1; D. J. H. Chung, B. Garbrecht, M. J. Ramsey-Musolf and S. Tulin, Phys. Rev. Lett. 102 (2009) 061301; K. Funakubo, S. Tao and F. Toyoda, Prog. Theor. Phys. 109 (2003) 415.

[12] M. Carena, G. Nardini, M. Quiros and C. E. M. Wagner, Nucl. Phys. B 812 (2009) 243.

[13] K. Funakubo and E. Senaha, Phys. Rev. D 79 (2009) 115024.

[14] M. Pietroni, Nucl. Phys. B 402 (1993) 27; S. J. Huber and M. G. Schmidt, Nucl. Phys. B 606 (2001) 183; K. Funakubo, S. Tao and F. Toyoda, Prog. Theor. Phys. 114 (2005) 369.

[15] A. Menon, D. E. Morrissey and C. E. M. Wagner, Phys. Rev. D 70 (2004) 035005; S. J. Huber, T. Konstandin, T. Prokopec and M. G. Schmidt, Nucl. Phys. B 757 (2006) 172.

[16] S. W. Ham, E. J. Yoo and S. K. OH, Phys. Rev. D 76 (2007) 075011; S. W. Ham and S. K. OH, Phys. Rev. D 76 (2007) 095018; A. Ahriche, S. Nasri, Phys. Rev. D83 (2011) 045032.

[17] C. -W. Chiang, E. Senaha, JHEP 1006 (2010) 030.

[18] J. Kang, P. Langacker, T. Li, T. Liu, JHEP 1104 (2011) 097.

[19] S. Kanemura, T. Shindou, K. Yagyu, Phys. Lett. B699 (2011) 258-263.

[20] A. Zee, Phys. Lett. B 93 (1980) 389 [Erratum-ibid. B 95 (1980) 461]; Phys. Lett. B 161 (1985) 141; Nucl. Phys. B 264 (1986) 99; L. M. Krauss, S. Nasri and M. Trodden, Phys. Rev. D 67 (2003) 085002;

[21] R. S. Gupta, J. D. Wells, Phys. Rev. D81 (2010) 055012; G. Marshall, M. Sher, Phys. Rev. D83 (2011) 015005; M. Aoki, S. Kanemura, T. Shindou, K. Yagyu, arXiv:1108.1356 [hep-ph].

[22] S. L. Glashow and S. Weinberg, Phys. Rev. D 15 (1977) 1958.

[23] V. D. Barger, J. L. Hewett, R. J. N. Phillips, Phys. Rev. D41 (1990) 3421; Y. Grossman, Nucl. Phys. B426 (1994) 355-384.

[24] M. Aoki, S. Kanemura, K. Tsumura, K. Yagyu, Phys. Rev. D80 (2009) 015017; S. Su, B. Thomas, Phys. Rev. D79 (2009) 095014; H. E. Logan, D. MacLennan, Phys. Rev. D79 (2009) 115022.

[25] Y. Okada, M. Yamaguchi, T. Yanagida, Prog. Theor. Phys. 85 (1991) 1-6; J. R. Ellis, G. Ridolfi, F. Zwirner, 
Phys. Lett. B257 (1991) 83-91; H. E. Haber, R. Hempfling, Phys. Rev. Lett. 66 (1991) 1815-1818.

[26] R. Harnik, G. D. Kribs, D. T. Larson, H. Murayama, Phys. Rev. D70 (2004) 015002. hep-ph/0311349.

[27] R. R. Parwani, Phys. Rev. D 45 (1992) 4695 [Erratumibid. D 48 (1993) 5965] [Phys. Rev. D 48 (1993) 5965].

[28] F. R. Klinkhamer, N. S. Manton, Phys. Rev. D30 (1984)
2212.

[29] P. B. Arnold, L. D. McLerran, Phys. Rev. D36 (1987) 581.

[30] R. Barbieri, L. J. Hall, V. S. Rychkov, Phys. Rev. D74 (2006) 015007. 